\title{
Analytical and Numerical Investigation of Noise in Nanoscale Ballistic Field Effect Transistors
}

\author{
Giuseppe Iannaccone
}

Dipartimento di Ingegneria dell'Informazione: Elettronica, Informatica, Telecomunicazioni, Università di Pisa 


\title{
Analytical and Numerical Investigation of Noise in Nanoscale Ballistic Field Effect Transistors
}

\author{
G. IANNACCONE \\ Dipartimento di Ingegneria dell'Informazione: Elettronica, Informatica, Telecomunicazioni, \\ Università degli studi di Pisa, Via Caruso, I-56122, Pisa, Italy \\ g.iannaccone@iet.unipi.it
}

\begin{abstract}
The current of ballistic nanoscale MOSFETs is expected to exhibit shot noise, essentially because the electron distribution is very far from equilibrium. Here, we derive an analytical expression of shot noise in fully ballistic MOSFETs and show how it can be computed on the basis of numerical simulations of the DC electrical properties. We show that the power spectral density of shot noise of the drain current is strongly suppressed as an effect of both Pauli exclusion and electrostatic interaction. The amount of such suppression depends on the device structure, and in particular on the gate capacitance. Results on shot noise of the gate current are also shown, since such the leakage current might be significant in nanoscale MOSFETs, for small equivalent oxide thickness.
\end{abstract}

Keywords: noise, ballistic transport, MOSFETs

\section{Introduction}

The issue of whether nanoscale MOSFETs with channel lengths close to $10 \mathrm{~nm}$ may exhibit ballistic transport is still debated, even if also the ITRS Roadmap [1] predicts that a significant component of the total current will be ballistic. As the ballistic limit is approached, noise in the channel should seamlessly move from thermal to shot. Here, we investigate noise in the limit of ballistic transport, and derive an expression of the shot noise power spectral density which is suppressed with respect to Poissonian noise because of Pauli and Coulomb interactions among electrons.

This topic is important not only because it represents one of the first cases in which effects typical of mesoscopic transport emerge also in "secular" devices, such as the MOSFETs, and at room temperature, but also because appropriate noise models of nanoscale MOSFETs are required by circuit designers, especially for analog and mixed-signal applications.

Here, we have derived an analytical model of the channel noise in the case of ballistic transport, that allows us to compute its power spectral density on the basis of numerical simulations of DC electrical properties.

Another significant shot noise component in nanoscale MOSFETs is provided by the gate current, which can be significant, for the predicted Equivalent Oxide Thicknesses of about $1 \mathrm{~nm}$. This component might also be significantly altered by the presence of native or stress-induced traps in the oxide that assist carrier tunneling, and suppress noise with respect to a Poissonian process [2]. Here we focus on StressInduced Leakage Currents (SILCs), for which experiments are available. However, the same effect should also occur in the case of trap-assisted-tunneling through native traps, that dominates transport through very thin oxides at small fields.

\section{Shot Noise of the Drain Current}

Shot noise in multimode ballistic conductors, a class of devices including MOSFETs, has been recently investigated in Refs. [3-6]. Suppression of shot noise has been predicted as a result of Fermi statistics at the source contact, and of electrostatic interaction in the 
channel. We derive a model based on parameters that can be extracted from a numerical simulation of the DC properties of a ballistic MOSFET [6].

We show that, in the range of operating gate-tosource and drain-to-source voltage, shot noise can be strongly suppressed with respect to that of a Poissonian process, at room temperature. Such suppression must be taken into account in accurate compact models of device noise to be used in circuit simulation.

For a given bias point, we can assume that the subband profile has been obtained from the self-consistent solution of the Poisson-Schrödinger equation in two dimensions [7]. Fluctuations of the drain current are only due to fluctuations of the occupancy of incoming states (Pauli interaction), and to potential fluctuations induced by the mentioned fluctuations of occupation factors through long-range electrostatic interactions. Both terms are expected to suppress current noise with respect to Poissonian shot noise.

In order to apply the model on a realistic structure, we take the $25 \mathrm{~nm}$ "Well tempered" MOSFET considered in Ref. [7], and make the simplifying assumption of fully ballistic transport. We verify that for all bias points we can reasonably assume that only the lowest doubly degenerate subband is occupied. From the simulation of the DC properties we can extract the maximum value of the first subband in the channel $E_{M}$ for any operating point. In Fig. 1 it is plotted as a function of the gate voltage for two different values of the drain to source voltage.

For brevity, let us use the following notation

$$
\langle\langle h\rangle\rangle \equiv 2 \int_{0}^{\infty} d E_{y} \int_{0}^{\infty} d E_{z} N_{2 D} h,
$$

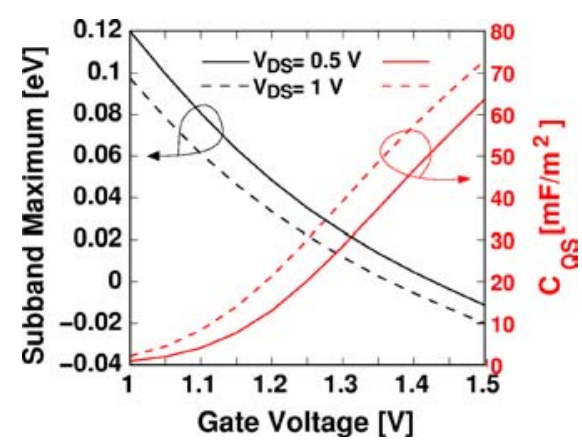

Figure 1. Maximum value of the subband energy in the channel $E_{M}$ and $C_{Q S}$ as a function of the gate voltage for two different drain voltages. for any function $h$ of the two kinetic energy components in the transport direction $E_{y}$, and in the transversal direction $E_{z}$ ( $x$ is the vertical direction). Here, $N_{2 D}$ is the $2 \mathrm{D}$ electron density in the subband.

The electron density per unit area in the point corresponding to the subband maximum is therefore

$$
\begin{aligned}
n_{2 D}= & \left\langle\left\langle f_{S}\left(E_{y}+E_{z}+E_{M}\right)\right.\right. \\
& \left.\left.+f_{D}\left(E_{y}+E_{z}+E_{M}\right)\right\rangle\right\rangle,
\end{aligned}
$$

where $f_{S}(E)$ and $f_{D}(E)$ are the Fermi Dirac occupation factors corresponding to the Fermi energy of the source $E_{F S}$ and of the drain $E_{F D}$, respectively. We assume that fluctuations of the propagating states occupation factor affect $n_{2 D}$ directly and through their electrostatic effect on $E_{M}$. From (1), we have

$$
\delta n_{2 D}=\left\langle\left\langle\delta f_{S}+\delta f_{D}\right\rangle\right\rangle+\delta E_{M}\left\langle\left\langle\frac{\partial f_{S}}{\partial E}+\frac{\partial f_{D}}{\partial E}\right\rangle\right\rangle .
$$

We use a very simple approximation for the electrostatics, synthesizing all electrostatic effects in a unique gate capacitance per unit area $C_{G}$, which gives us the relation between the electron density and the subband potential at the maximum:

$$
\delta E_{M}=q^{2} \delta n_{2 D} / C_{G},
$$

from which we obtain

$$
\delta E_{M}=\frac{q^{2}\left\langle\left\langle\delta f_{S}+\delta f_{D}\right\rangle\right\rangle}{C_{G}+C_{\mathrm{QS}}+C_{\mathrm{QD}}},
$$

where we have defined

$$
C_{\mathrm{QS}} \equiv q^{2}\left\langle\left\langle\frac{\partial f_{S}}{\partial E_{F S}}\right\rangle\right), \quad C_{\mathrm{QD}} \equiv q^{2}\left\langle\left\langle\frac{\partial f_{D}}{\partial E_{F D}}\right\rangle\right) .
$$

The ballistic current density I, computed at the subband maximum, is given by

$$
\begin{aligned}
I= & q\left\langle\left\langlev _ { y } \left[ f_{S}\left(E_{y}+E_{z}+E_{M}\right)\right.\right.\right. \\
& \left.\left.\left.-f_{D}\left(E_{y}+E_{z}+E_{M}\right)\right]\right\rangle\right\rangle,
\end{aligned}
$$

where $v_{y}=\sqrt{2 E_{y} / m_{t}}$ is the velocity in transport direction. Again, fluctuations of $f_{S}$ and $f_{D}$ act directly on $I$ and through long range electrostatic interactions (through $E_{M}$ ). For simplicity of notation, we now consider the far from equilibrium case, in which 
$f_{D} \ll 1$ and can be discarded (generalization for quasiequilibrium is trivial).

$$
\delta I=q\left\langle\left\langle v_{y} \delta f_{S}+v_{y} \delta E_{M} \frac{\partial f_{S}}{\partial E}\right\rangle\right\rangle .
$$

If we substitute (5) into (8), after straightforward derivation, we obtain

$$
\delta I / q=\left\langle\left\langle v_{y}\left(1-\frac{\tilde{v}_{S} C_{\mathrm{QS}}}{C_{G}+C_{\mathrm{QS}}} \frac{1}{v_{y}}\right) \delta f_{S}\right\rangle\right\rangle,
$$

where $\tilde{v}_{S}$ is a weighted average of the velocity $v_{y}$ :

$$
\tilde{v}_{S} C_{\mathrm{QS}}=-q^{2}\left\langle\left\langle v_{y} \frac{\partial f_{S}}{\partial E_{F S}}\right\rangle\right) .
$$

Considering the current as a sum of pulses each carrying charge $q$, that $\overline{\delta f_{S}^{2}}=f_{S}\left(1-f_{S}\right)$, and that the occupation factor of different modes are uncorrelated, we can write the noise suppression factor (Fano factor) as

$$
\begin{aligned}
\gamma & =\frac{S}{2 q I} \\
& =\frac{\left\langle\left\langle v_{y}\left(1-\frac{\tilde{v}_{S} C_{\mathrm{QS}}}{C_{G}+C_{\mathrm{QS}}} \frac{1}{v_{y}}\right)^{2} f_{S}\left(1-f_{S}\right)\right\rangle\right\rangle}{\left\langle\left\langle v_{y} f_{S}\right\rangle\right\rangle} .
\end{aligned}
$$

In Eq. (11) we can quickly recognize the two contributions to noise suppression of Pauli exclusion (the term $f_{S}\left(1-f_{S}\right)$ ) and of Coulomb coupling (the term squared between round parentheses). In Fig. 1 the term $C_{\mathrm{QS}}$ is plotted as a function of the gate voltage, while in Fig. 2 we plot the Fano factor as a function of the gate voltage for $V_{\mathrm{DS}}=1 \mathrm{~V}$, also considering the separate effects of Pauli exclusion, of Coulomb coupling, as computed from (11).

As can be seen in Fig. 2, Coulomb coupling is predominant, since for the device and bias conditions considered the electron gas is not degenerate, and shot noise suppression may be significant with respect to a purely poissonian process.

\section{Shot Noise of the Gate Current}

Shot noise in the gate current has been previously investigated by the author, and a model has been developed for transport and noise in the presence of an arbitrary distribution of traps assisting tunneling [2]. Recently, we have been able to use such model for extracting the

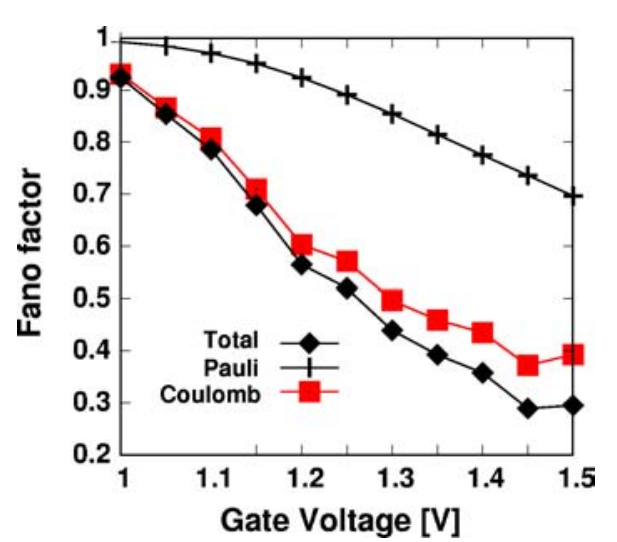

Figure 2. Fano factor of the channel shot noise as a function of the gate voltage for $V_{\mathrm{DS}}=1 \mathrm{~V}$, for the $25 \mathrm{~nm}$ "Well Tempered" MOSFETs. Effect of only Pauli exclusion, only Coulomb Interaction, and of both.

energy distribution of traps, by fitting numerical simulations to experiments on stressed MOS capacitors. The reader interested in the details of the method can find them in Ref. [8]. Here, we just want to show that a Gaussian distribution of traps in the silicon oxide, centered $0.5 \mathrm{eV}$ below the energy corresponding to silicon conduction band edge and with standard deviation of $82 \mathrm{meV}$, is sufficient to obtain a very good fitting of both DC electrical properties and noise properties, as shown in Fig. 3, where results are shown for a $6 \mathrm{~nm}$ MOS capacitor.

For much thinner oxides, such as those used in nanoMOSFETs, SILCs are typically much smaller than direct or Fowler-Nordheim tunneling currents. However, for very thin oxides at small-moderate fields, a dif-
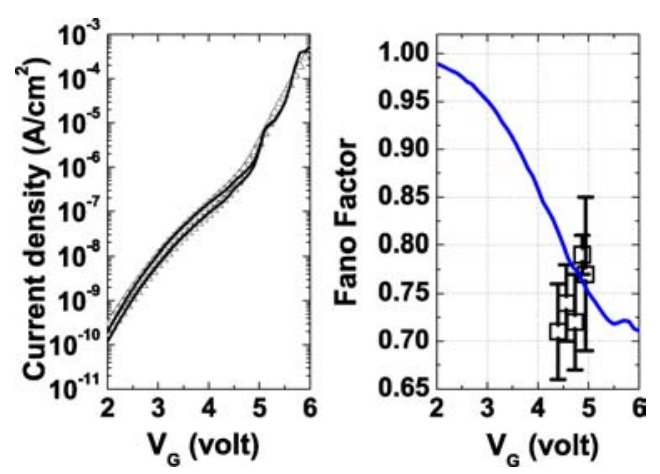

Figure 3. Left: theoretical(solid lines) and experimental (triangles) I-V characteristics of a MOS capacitor with 6 nm oxide. Right: theoretical (solid lines) and experimental (symbols) fano factor of the same MOS structures. Experiments are taken from Ref. [2]. 
ferent current component, due trap-assisted-tunneling through native traps, is dominant. Even if noise measurements are not available for such case, we believe that the same modeling approach should be applied, and that a noise suppression similar to that obtained in the case of SILCs is to be expected.

\section{Conclusion}

We have derived an expression for the shot noise of the drain current with two well-identified contributions from Pauli exclusion and Coulomb repulsion. A simple interpretation has been given, in terms of a "gate" capacitance and of quantum capacitances. Detailed numerical calculation is straightforward, once subband profiles are computed with a 2D Poisson-Schroedinger solver. From the application point of view, particular care has to be devoted to the resistance of source and drain extensions, since the associated thermal noise is expected to make a significant contribution to the channel noise.

As far as the noise of the gate current is concerned, we have developed a model that takes into account trapassisted tunneling through traps caused by electric field stress and through native traps. For fresh oxides a Poissonian shot noise model is valid, while we have shown that for stressed oxides, such as those of flash mem- ories after a few program/erase cycles, a suppressed Fano factor is obtained, typically between 0.7 and 0.8 . Similar results is expected for fresh very thin oxides in the presence of native traps.

\section{Acknowledgments}

This work has been supported by the FIRB Project "Sistemi miniaturizzati per elettronica e fotonica" and by the EU through the SINANO Network of Excellence.

\section{References}

1. International Technology Roadmap for Semiconductors 2003, Semiconductor Industry Association, S. Josè, USA (http://public.itrs.net).

2. G. Iannaccone, F. Crupi, B. Neri, and S. Lombardo, IEEE Trans. Electron Dev., 50, 1363 (2003).

3. Y. Naveh, A.N. Korotkov, and K.K. Likharev, Phys. Rev. B, 60, R2169 (1996).

4. O.M. Bulashenko and J.M. Rub, Phys. Rev. B, 66, 045310 (2002).

5. O.M. Bulashenko and J.M. Rub, Phys. Rev. B, 65, 045307 (2001).

6. G. Gomila, I.R. Cantalapiedra, T. González, and L. Reggiani Phys. Rev. B, 66, 075302 (2002).

7. G. Fiori and G. Iannaccone, Appl. Phys. Lett., 81, 3672 (2002).

8. A. Nannipieri, G. Iannaccone, and F. Crupi, Microelectronics Reliability, to be published, 2004. 